\title{
Telehealth: Synopsis
}

\section{Paul Butel}

THE COVID-19 PANDEMIC has changed the Australian medical landscape, especially in relation to how we need to provide clinical care in general practice. The recent federal Medicare Benefits Schedule changes have opened up clinical telehealth to general practice. It is going to be an increasingly used clinical modality, particularly as hospitals move their resources and focus elsewhere, and there is a need to provide ongoing care to our patients.

The telehealth modality bridges the intersecting need for general practice care with that of the patient's social isolation. This new adaptation can initially be organisationally daunting. However, telehealth has been deployed and established in other clinical contexts (eg remote mental health services), as well as in some general practices across Australia. ${ }^{1-8}$ The Royal Flying Doctor Service operating out of its traditional bases in Queensland is one such telehealth primary care provider within this clinical context. ${ }^{9}$

The telehealth consult shares many similar clinical elements to that of the traditional face-to-face general practice consult. ${ }^{10}$ The telehealth consult still has a structure and clinical flow. ${ }^{11}$ There are, however, some important elements or factors to be aware of in this new consult paradigm.

The important elements to remember to incorporate include the following:

- The consult requires the clarification of the patient's details.

- It is essential to re-establish contact with the patient if the consult is interrupted or for follow-up. These details need to be confirmed at the initial preliminary phase, before moving to the critical clinical stage of the consult. These details can be obtained by the reception staff at the time of the consult's booking or by the clinician at the beginning of the consult.

- The setting for the telehealth consult needs to have the same degree of privacy as a regular face-to face-consult. This applies to the settings of both the patient and the clinician.

- The patient needs to confirm that they consent to this modality of consult.

- The telehealth consult is heavily weighted towards the patient's presenting complaint and history.

- The components of the examination are often minimal and usually restricted to that of general observations.

- The examination may be supplemented by audio-visual material or digital imagery supplied by the patient.

- Traditional clinical measures of observation such as oxygen saturations, heart rate, blood pressure, temperature and blood sugar may be technically unavailable.

- These absences need to be acknowledged and factored into your formulation of a potential diagnosis (recognising that it often broadens that differential).

- A detailed discussion with the patient about your diagnostic impression and reasoning, as well as other significant potential differentials, is essential.

- It empowers your physically isolated patient and facilitates their compliance with your advised management plan.

- Communication, as with all consultations, is the underlying foundation of the telehealth consult.
- The consult is a shared-care patient clinical engagement.

- Where possible, any advice to the patient should be delivered with a 'closed-loop' communication technique. $^{12}$

- Encouraging the patient to query the plan minimises the chance of misinterpretation.

- Any management plan needs to consider the situational restrictions and logistical options available to the patient.

- Where there is clinical uncertainty, this should be openly acknowledged.

- A discussion about realistic triggers and processes for follow-up needs to be clearly outlined. It can be supplemented with (de-identified) written advice, which can be delivered by an agreed available communication medium. ${ }^{13}$

- Digital media and devices can aid the assessment, as well as facilitate the delivery of care and management.

- Finally, the documentation in your clinical medical record should

be detailed and reflective of the consultation.

- It is essential to document that the consult was a telehealth consult.

- It is essential to have a provisional diagnosis and a differential, which willl aid you later with any subsequent reappraisal or review of the patient's progress.

- Any clinically critical relative negative findings or limitations should be noted in the documentation.

As the patient's usual general practitioner, you have the advantage of continuity of care; you will already have established rapport and a more detailed understanding of their situation. This is reassuring and empowering to both parties of the consult. It is important also to be aware that the 
patients are willing users of this type of care delivery. Like you, they are also mindful of its limitations.

\section{First published online 28 April 2020.}

\section{Author}

Paul Butel FACRRM, M Med Av, Dip Aero RT, PG Clin Epi, PG Occ Med, MB.ChB, BSc, Telemedicine Consultant and Rural General Practitioner, The Royal Flying Doctor Service of Australia - Queensland Section (Mt Isa), Qld. pbutel@rfdsqld.com.au Competing interests: None.

Provenance and peer review: Commissioned, peer reviewed.

Citation: Butel P. Telehealth: Synopsis. Aust J Gen Pract 2020;49 Suppl 16. doi: 10.31128/AJGPCOVID-16.

\section{References}

1. Australian College of Rural and Remote Medicine. ATHAC telehealth standards framework ACRRM telehealth guidelines. Brisbane: ACRRM, 2016.

2. Australian College of Rural and Remote Medicine. eHealth. Brisbane: ACRRM, 2020. Available at www.ehealth.acrrm.org.au [Accessed 25 April 2020].

3. Brown LJ, Jones GM, Bond MJ. E-health: Psychosocial challenges for South Australian rural mental health consumers. Rural Remote Health 2019;19(3):5103. doi: 10.22605/RRH5103.

4. Meurk C, Leung J, Hall W, Head BW, Whiteford H. Establishing and governing e-mental health care in Australia: A systematic review of challenges and a call for policy-focussed research. J Med Internet Res 2016;18(1):e10. doi: 10.2196/jmir.4827.

5. Nicholas J, Huckvale K, Larsen ME, Basu A, Batterham PJ, Shaw F, et al. Issues for eHealth in psychiatry: Results of an expert survey. J Med Internet Res 2017;19(2):e55. doi: 10.2196/jmir.6957.

6. McKenzie R, Williamson M. The league of extraordinary generalists: A qualitative study of professional identity and perceptions of role of GPs working on a national after hours helpline in Australia. BMC Health Serv Res 2016;16:142. doi: 10.1186/s12913-016-1387-5.

7. Ayton J. Australian Antarctic medical caregeneralist scope of practice, training and support - A space analogue. In: Medical Care for Humans in Space. 68th International Astronautical Congress 2017. 26 September 2017. Paris: International Astronautical Federation (IAF), 2017. p. 1978-84.

8. Abbott LM, Miller R, Janda M, et al. A review of literature supporting the development of practice guidelines for teledermatology in Australia. Australas J Dermatol 2020. doi: 10.1111/ajd.13249.

9. Cherry JJ, Rich WC, McLennan PL. Telemedicine in remote Australia: The Royal Flying Doctor Service (RFDS) medical chest program as a marker of remote health. Rural Remote Health 2018;18(4):4502. doi: 10.22605/RRH4502.

10. Downes MJ, Mervin MC, Byrnes JM, Scuffham PA. Telephone consultations for general practice: A systematic review. Syst Rev 2017;6(1):128. doi: 10.1186/s13643-017-0529-0.

11. The Royal Australian College of General Practitioners. Telehealth video consultations guide. East Melbourne, Vic: RACGP, 2019.

12. Smith K. Effective communication with primary care providers. Pediatr Clin North Am 2014;61(4):671-79. doi: 10.1016/j.pcl.2014.04.004.
13. The Royal Australian College of General Practitioners. Templates and factsheets. East Melbourne, Vic: RACGP, 2020. Available at www. racgp.org.au/running-a-practice/technology/ clinical-technology/telehealth/templates-andfactsheets [Accessed 25 April 2020]. 